\title{
Existence of High Frequency Electricity at Acupoints and Meridian Flow Observations via the Frequency Spectrum
}

\author{
Taeho Son ${ }^{1, *} \cdot$ Moonhee Lee ${ }^{1} \cdot$ Choolhyeong Rhee $^{2} \cdot$ Namsoo Kim $^{2}$
}

\begin{abstract}
We revealed the existence of high frequency ( $\mathrm{HF}$ ) electricity flowing through the meridians known to oriental medicine by observing these signals at various acupoints on the human body. This was clearly and scientifically demonstrated by recording the frequency spectrum at these acupoints using a spectrum analyzer. The analysis of the measured spectrum found in 5 particular acupoints of each of the 12 meridians showed that a flow exists in these acupoints that could be measured with a spectrum frequency between $1 \mathrm{MHz}$ and 80 $\mathrm{MHz}$ and an intensity of -70 to $-60 \mathrm{dBm}$. Since the presence of these spectrums can be equated with the presence of electricity, we can emphatically conclude that high frequency electricity exists in humans and flows through the acupoints used in oriental medicine. The same characteristic frequency spectrums were found at all the acupoints along the same meridian and were shown to have the same type of HF current flow. In this way, we were able to confirm the existence of meridian flow in a scientific manner. In addition, it was found that as the distance from the organ increased, the intensities of the spectrum measured on that meridian decreased, which is exactly the behavior electrical transmission line theory would predict.
\end{abstract}

Key Words: Acupoint, Acupuncture, Frequency Spectrum, HF Electricity, Meridian.

\section{INTRODUCTION}

The meridian system in acupuncture and moxibustion $(\mathrm{A} \& \mathrm{M})$ medicine has great significance in traditional Chinese medicine (TCM) and is one of its most fundamental theories. These techniques have had a history of use of 5 millennia in East Asia. Since ancient times scholars in this part of the world have seen the human body and nature as one, an idea related to the concept of Yin and Yang. Any health issue is thought to be the result of an unbalanced Yin-Yang, and as such, the result and role of medicine is to rebalance this Yin-Yang through various means. These ideas about balance and connectedness lead to theories about information lines that connect the materials constituting the human body, therefore, it is important to identify and reinforce the existence of these lines. In TCM the basic substances composing the human body are said to include the $Q i$ (which could be translated as bioenergy), blood and essence. As a part of TCM external medicine, the function of A\&M is often to extract unbalanced $Q i$ from the patient's body through certain, specific acupuncture points (acupoints) that exist on the body's "meridian". Depending on the patient's complaint, the

Manuscript received September 4, 2019 ; Revised November 28, 2019 ; Accepted January 13, 2020. (ID No. 20190904-073J)

${ }^{1}$ Department of IT Engineering, Soonchunhyang University, Asan, Korea

${ }^{2}$ Academy of Korean Authentic Acupuncture and Moxibustion, Seoul, Korea

"Corresponding Author: Taeho Son (e-mail: thson@sch.ac.kr)

This is an Open-Access article distributed under the terms of the Creative Commons Attribution Non-Commercial License (http://creativecommons.org/licenses/by-nc/4.0) which permits unrestricted non-commercial use, distribution, and reproduction in any medium, provided the original work is properly cited.

(c) Copyright The Korean Institute of Electromagnetic Engineering and Science. All Rights Reserved. 
TCM practitioner will determine the appropriate acupoints to use. Therefore, we can think of A\&M medicine as an optimized stimulation therapy through which the body balances itself [16]. The meridian system is the circulation system for $Q i$; it consists of lines that vertically and horizontally wrap around the body and connect our internal organs to the body surface. There is a constant flow of $Q i$ passing through the meridian of a healthy individual. The acupoints on the body surface are points on this meridian, and these points can collect and store $Q i$ cycling in from internal organs. These points react when the body has a disease but can also be used as preventive and treatment points for disease. An acupoint is also referred to as a biological action point (BAP). There are 361 acupoints present on the meridian, based on the standard proposed by the World Health Organization (WHO). To find these points exactly, we use either a proportional measurement to calculate the position using the skeleton as a reference or we use finger measurements in which the width of the finger is used as a reference distance from a landmark spot. An investigation that used acupoints identified with an arrow found that acupuncture and moxibustion are more effective when applied to the correct, traditional acupoints. Unfortunately, finding the somewhat subjective position of acupoints is unscientific. Due to the debate surrounding the existence of certain acupoints, and how scientific the treatment is, most countries currently use acupuncture as a form of complementary and alternative medicine (CAM) in tandem with conventional treatment. A\&M is even used in the armed forces of some countries. Numerous research papers have discussed the effect and mechanisms of acupuncture.

To scientifically explain the effects of acupuncture, we first need to investigate the characteristics of the human meridian and acupoints. Studies have been performed on electrical properties found at the meridian and acupoints. Conduction current $[2,7-10]$ and electrodermal characteristics [11-16] have all been measured at the acupoints. Although these have produced interesting results, acupoints and the concept of meridians have yet to be characterized in a scientific, measurable manner. All such attempts to do so have been based on looking at DC (direct current) or low frequency range $(3-5 \mathrm{kHz})$ electrical signals. Studies to determine the operation of acupoints as electrical circuit components have also been undertaken. Skin resistance, conductance, impedance, susceptance, bio-electric potential and DC voltage at acupoints and on the meridians have all been measured [4,17-26]. The values and characteristics expressed by circuit elements have yet to sufficiently explain the effect of $\mathrm{A} \& \mathrm{M}$ or the flow of the meridian system on the human body. Other researchers have looked at the effects or color phenomena between meridians using special cameras in the infrared or visible ranges [27-31]. Meridians have been represented using an impedance matching and mismatching model based on the transmission line theory from electrical engineering, and have also been expressed using a mesh reticular model $[32,33]$.

In this study, we present evidence showing the existence of high frequency (HF) electricity at human acupoints. This is an important step toward understanding the meridian flow system that has been fundamental to oriental medicine since ancient times. The presence of HF electricity was measured using a spectrum analyzer and each meridian described according to its own frequency spectrum. By comparing the measured spectrum in the same meridian, the meridian flow that is the basis of present day oriental medicine and is described in ancient literature is proven to exist. The measured spectrum intensities show that $Q i$ originates from the corresponding organ and is transmitted according to the characteristics of transmission line theory in electrical engineering. Due to the very small spectrum intensity at acupoints, all measurements were taken in an anechoic shielding room to be exact and avoid external noise. The equipment used was a FSH6 spectrum analyzer (100 kHz to $6 \mathrm{GHz})$ manufactured by Rohde \& Schwarz, Munchen, Germany. The 0.2 $\mathrm{mm} \times 50 \mathrm{~mm}$ (diameter $\times$ length) stainless steel needles connected with an alligator clip to a BNC radio Frequency (RF) cable were used for the spectrum measurements. HF spectrum were measured at 5 particular acupoints on 12 meridians in both male and female test subjects. Measurements for the meridian flow were taken in the main acupoints of the same 12 meridians. The spectrum measurement method was verified in two previous studies [34, 35], and this paper was prepared by combining those two pieces of research.

This paper presents, in Section II, the measurement of the HF spectrum and in Section III the spectrums are shown to be different when the meridians are different. In Section IV, we reveal the meridian flow.

\section{MEASUREMENT OF HF SPECTRUM AT ACUPOINT}

In this study, HF spectrums at acupoints on the meridian are measured using a spectrum analyzer. A total of 60 points of 5 types are tested: primary point, cleft point, connecting point, front alarm point, and a back associated point on the 12 meridians, e.g., from the lung meridian of hand-Taiyin to the liver meridian of foot-Jueyin. A BNC RF cable is used to connect the needle and spectrum analyzer. These particular acupoints are commonly used in acupuncture for various kinds of disease treatment. A Dongbang needle made from stainless steel with a $0.2 \mathrm{~mm} \times 50 \mathrm{~mm}$ (diameter $\times$ length) is used to perform the measurements.

To remove unwanted external noise from the data, we took all measurements in a room with anechoic shielding. This kind of anechoic shielding room is generally used to measure antenna radiation characteristics [36] and electromagnetic interference 
(EMI). The anechoic shielding room used for the measurements is shown in Fig. 1. The specification for frequency range of the anechoic room is from $400 \mathrm{MHz}$ to $6 \mathrm{GHz}$. This frequency specification is the frequency ranges for anti-reflection by the electromagnetic wave absorption. In accordance with IEEE299 regulation, the anechoic shield room used in this study has a shielding effectiveness of $110 \mathrm{~dB}$ up to $100 \mathrm{MHz}$.

Fig. 2 shows two spectrums measured inside and outside the anechoic shielding room. The measurements were taken by connecting a BNC cable to the spectrum analyzer and attaching an alligator clip to the center of the needle handle.

As shown in Fig. 2(a), there is a wide-band noise spectrum of frequency on $20-40 \mathrm{MHz}$ and $60-80 \mathrm{MHz}$ that is introduced when measurements are recorded outside the anechoic shielding room. As such, any measurements taken in such conditions might introduce an error in the frequency spectrum of the human acupoints, and this measurement data then becomes unre-

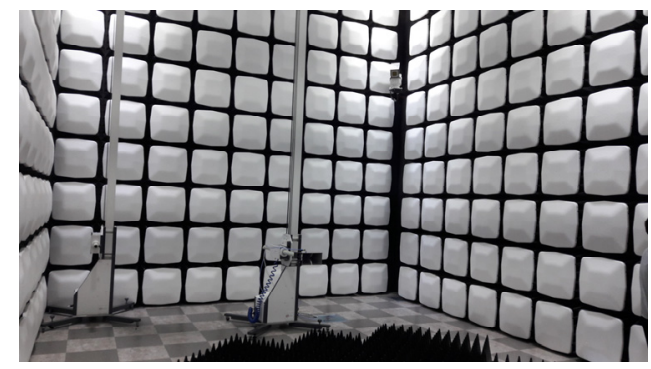

Fig. 1. Anechoic shielding room where measurements were taken.

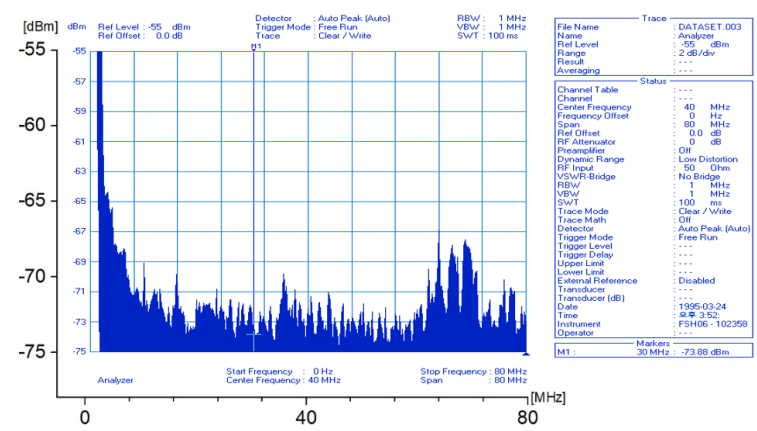

(a)

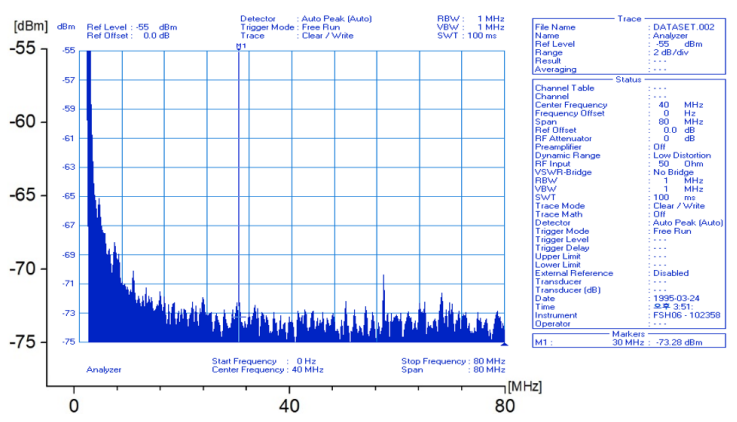

(b)

Fig. 2. Frequency spectrum outside (a) and inside (b) of the anechoic shielding room. liable. However, the interior of the room is free from noise, as shown in Fig. 2(b).

The measurements were made from acupoints on both male and female subjects. The man and woman tested were 61 years old, $75 \mathrm{~kg}$ and 63 years old, $58 \mathrm{~kg}$, respectively. The results of our analysis will be presented in Sections III and IV.

Fig. 3 shows the measured spectrum at SI4, a primary acupoint of the small intestine meridian of hand-Taiyang. SI 4 is the WHO standard acupuncture point location for Wangu (or wrist bone in English) [37]. Fig. 3(a) and (b) are for a male and a female, respectively. The detailed spectrum strengths versus frequencies are $-51.5 \mathrm{dBm}\left(7 \times 10^{-9} \mathrm{~W}\right)$ in the $1-5 \mathrm{MHz}$ range, -57 $\mathrm{dBm}\left(2 \times 10^{-9} \mathrm{~W}\right)$ at $13 \mathrm{MHz},-59 \mathrm{dBm}\left(1.3 \times 10^{-9} \mathrm{~W}\right)$ at 24 $\mathrm{MHz},-61 \mathrm{dBm}\left(7.9 \times 10^{-10} \mathrm{~W}\right)$ at $30 \mathrm{MHz},-65 \mathrm{dBm}(3.2 \times$ $\left.10^{-10} \mathrm{~W}\right)$ at $40 \mathrm{MHz},-68 \mathrm{dBm}\left(1.6 \times 10^{-10} \mathrm{~W}\right)$ at $60 \mathrm{MHz}$, and $-69 \mathrm{dBm}\left(1.3 \times 10^{-10} \mathrm{~W}\right)$ at $70 \mathrm{MHz}$. The spectrum shows various strengths in $1-80 \mathrm{MHz}$ frequency range. In the case of the female subject in Fig. 3(b), a similar spectrum to the man is found up to $20 \mathrm{MHz}$, but it is completely different beyond a frequency of $20 \mathrm{MHz}$. The spectrum strengths in Fig. 3 are relative values. For the absolute values of strength, we must consider the impedance matching with the ohmic losses for the BNC clip and needle. Since this study compares the trends of the measured frequency spectrums, the relative values of strength may be sufficient. However, as described above, we can see various $\mathrm{HF}$ spectrums ranging from $1 \mathrm{MHz}$ to $80 \mathrm{MHz}$ meas-

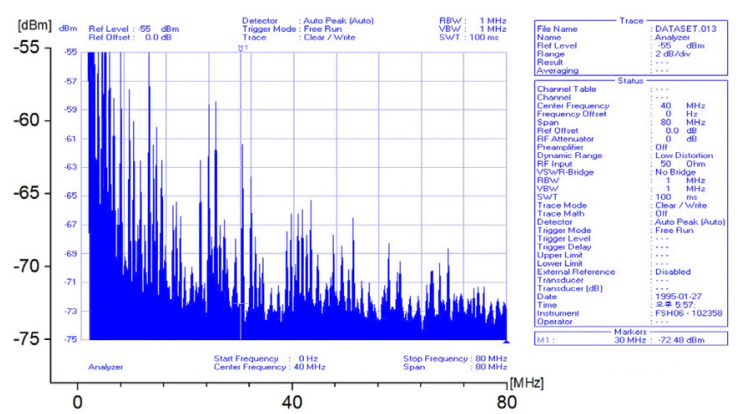

(a)

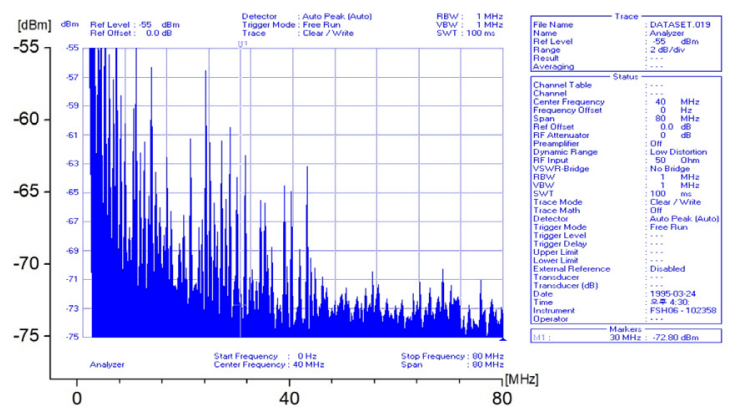

(b)

Fig. 3. Measured frequency spectrum at a primary point (SI4) on small intestine meridian of hand-Taiyang for a male (a) and a female (b). 
ured at the acupoints, as well as a very complicated HF electricity with various frequency components.

\section{DIFFERENT FREQUENCY SPECTRUMS BETWEEN THE MERIDIANS}

To investigate the frequency spectrum characteristics between the meridians, we compared the spectrums of the 60 pieces of measured frequency spectrum data. First, the spectrum at each primary acupoint of the 3 Yin meridians on the hand are shown in Fig. 4. More specifically, the lung meridian of hand-Taiyin, heart meridian of hand-Shaoyin, and pericardium meridian of hand-Jueyin were measured. The peak portion of each spectrum is represented by a dashed line for easy comparison.

Fig. 4(a) shows the frequency spectrum at LU9 on the primary acupoint of the lung meridian of hand-Taiyin. It has a wide band spectrum at frequencies of $1-20 \mathrm{MHz}$ and $25-45 \mathrm{MHz}$, and there is no apparent spectrum over a frequency of $60 \mathrm{MHz}$. On the other hand, the spectrum of HT7, which is the primary acupoint of the heart meridian of hand-Shaoyin, has a narrow frequency bandwidth at $1-10,15,25,30,40,60$, and $70 \mathrm{MHz}$, as shown in Fig. 4(b). Fig. 4(c) shows the spectrum of PC7, the primary acupoint of the pericardium meridian of hand-Jueyin. It is different compared to the spectrum for LU9 and HT7. Therefore, the spectrums of the three acupoints found on the inner wrist that are within $2 \mathrm{~cm}$ of each other can be seen to be quite different. These remarkable differences are ultimately due to differences in the meridian, which is demonstrated in the next section by showing that the frequency spectrum of the acupoints of the same meridian have the same or a similar spectrum. While the 3 Yin meridians of the hand are found on the inside of the arm and hand, the 3 Yang meridians of the hand are found on the outside of the arm and the hand.

Fig. 5 shows the measured frequency spectrum at each primary acupoint for the 3 Yang meridians of the hand.

The frequency spectrum at LI4, which is the primary acupoint of the large intestine meridian of hand-Yangming is shown in Fig. 5(a). It has a wide frequency band and a large intensity both at 1-15 MHz and 30-40 MHz. However, the frequency spectrum at SI4 shown in Fig. 5(b) has a narrower band and a lower intensity, around $40 \mathrm{MHz}$ lower than that seen for LI4. SI4 is the acupoint of the small intestine meridian of hand-Taiyang. Fig. 5(c) shows the frequency spectrum at TE4, which is the primary acupoint of the Sanjiao meridian of hand-Shaoyang; we can see it has a narrower band than LI4 and SI4. Like the 3 Yin meridians, we can see the 3 Yang meridians show quite different spectrum characteristics despite the fact that they are close to each other. They show these different HF electrical characteristics due to their locations on different meridians.

The $Q i$ from the meridian of hand-Yin to the meridian of hand-Yang passes down to the meridian of foot-Yin through each organ. Fig. 6 shows the measured frequency spectrum of each primary acupoint on the 3 Yin meridians of the foot.

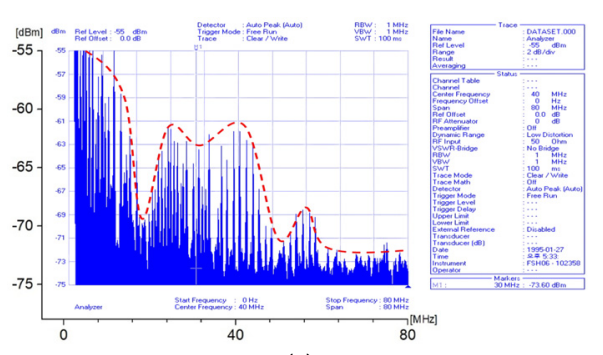

(a)

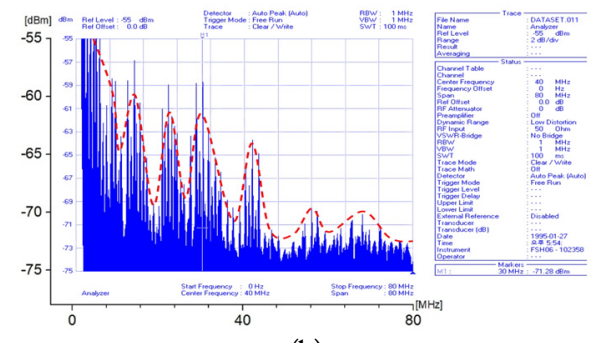

(b)

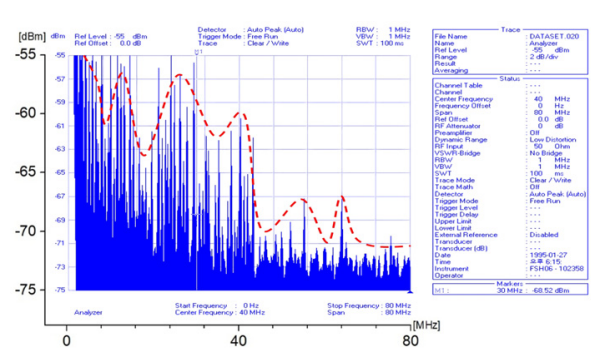

(c)

Fig. 4. Frequency spectrum on (a) LU9, (b) HT7 and (c) PC7. The red dashed lines in this and all subsequent frequency spectrum figures are the spectrum envelopes, which are the maximum connection lines of the spectrum strength.

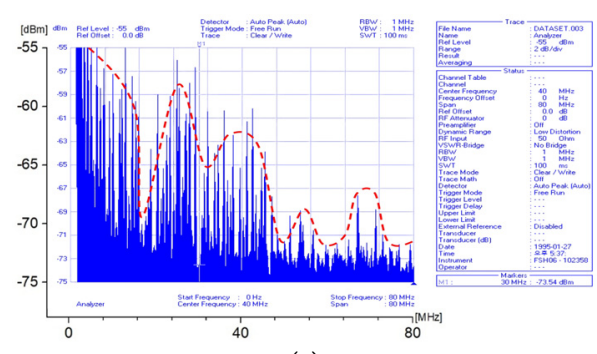

(a)

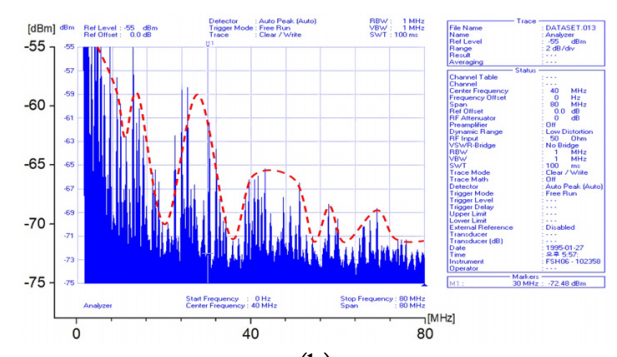

(b)

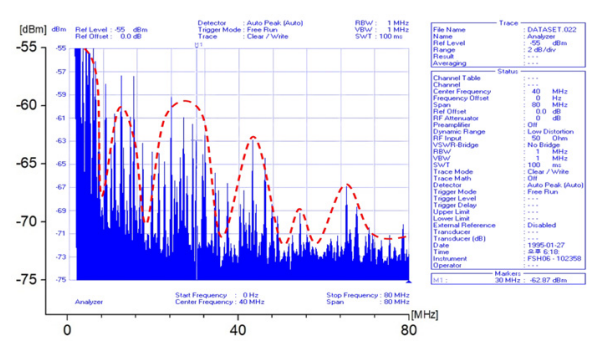

(c)

Fig. 5. Frequency spectrum on (a) LI4, (b) SI4, and (c) TE4. 


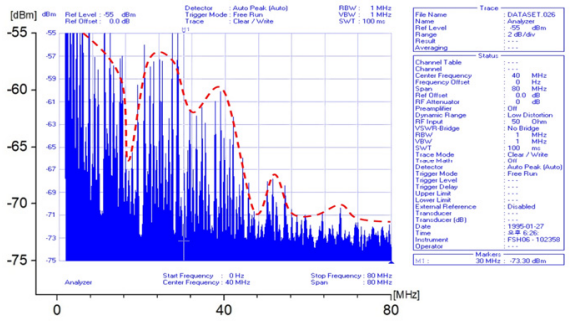

(a)

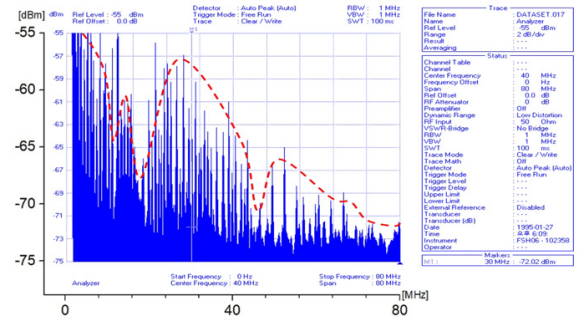

(b)

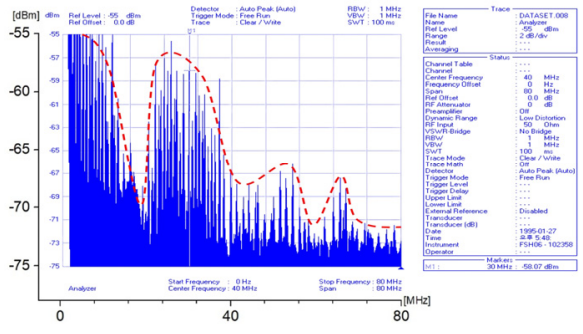

(c)

Fig. 6. Frequency spectrum on (a) SP3, (b) KI3, and (c) LR3.

The frequency spectrum at SP3, which is the primary acupoint of the spleen meridian of foot-Taiyin, is shown in Fig. 6(a). In the frequency band of 20-60 MHz, Fig. 6(a) is different than the spectrum at KI3 in Fig. 6(b) and LR3 in Fig. 6(c), which are the primary acupoints at the kidney meridian of foot-Shaoyin and the liver meridian of foot-Jueyin, respectively. Even at 70 $\mathrm{MHz}$, the spectrum positions and intensities of the three acupoints are different, showing a clear difference in the type and intensity of the HF electricity flowing through them.

Finally, let's look at the frequency spectrum of the 3 Yang meridians of the foot. Fig. 7 shows the frequency spectrums at ST42, BL64, and GB40, which are the primary acupoints of the stomach meridian of foot-Yangming, bladder meridian of footTaiyang, and gallbladder meridian of foot-Shaoyang, respectively.

The frequency spectrum in Fig. 7(a) is narrow with high intensity characteristics at frequencies of $1-5,10,25$, and $40 \mathrm{MHz}$. It is wide with low intensity at $60 \mathrm{MHz}$. For BL64 in Fig. 7(b), we can see a wide bandwidth. GB40 is characterized by a narrow in the low frequency band and a narrow but high intensity spectrum from $50-60 \mathrm{MHz}$.

As described above, in the cases of the 3 Yin meridians of the hand, the 3 Yang meridians of the hand, the 3 Yin meridians of the foot and the 3 Yang meridians of the foot, all have a frequency spectrum characteristics that are quite different. This means that $Q i$, in the form of $\mathrm{HF}$ electricity, flows through the human body, and the characteristics of this electricity, such as the waveforms and the frequency spectrum, are different for each of the meridians.

\section{REVEALING THE MERIDIAN FLOW PATH}

To reveal the mechanism of acupuncture therapy, it is first necessary to determine which energy exists at the acupoints and also confirm the phenomenon of meridian flow. In this study's previous chapter, we have shown by measurement with a spectrum analyzer in an anechoic shielding room that HF electricity exists at the acupoints. The existence of meridians will be addressed in this chapter. In the previous chapter, we saw that the frequency spectrum found on one meridian differs from those of the other meridian's acupoints. If the frequency spectrum at the acupoints on the same meridian are the same or similar to each other, they may have the same electrical characteristics. This means that the existence of the meridian is proven by HF electricity in the meridian, and by comparison of the frequency spectrum.

Fig. 8 shows the acupoints and pathways of the lung meridian of hand-Taiyin. The measured frequency spectrum from Zhongfu (LU1), the first acupoint on the lung meridian of hand-Taiyin, to Shaoshang (LU11), the last, are shown in Fig. 9.

The lung meridian of hand-Taiyin has 11 acupoints, and it flows toward the fingers in both the left and right arm from the lungs. While measuring in the left arm, the 4th acupoint, LU4, was skipped due to it being so near to the location of the 3rd acupoint, LU3. The important acupoints of the lung meridian of hand-Taiyin are Zhongfu (LU1) of the front alarm point, Chize (LU5), Kongzui (LU6) of the cleft point, Lieque (LU7) of the connecting point, Jingqu (LU8), Taiyuan (LU9) of the primary

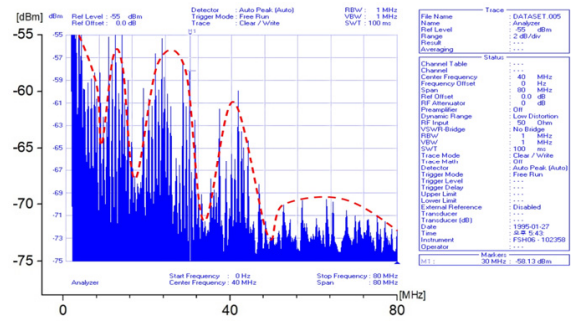

(a)

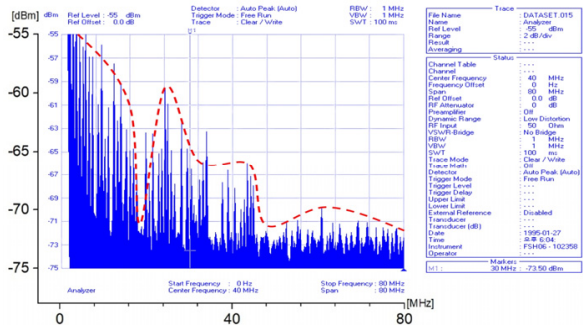

(b)

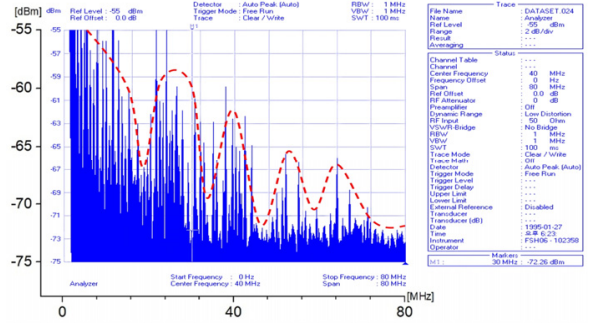

(c)

Fig. 7. Frequency spectrum on (a) ST42, (b) BL64, and (c) GB40. 


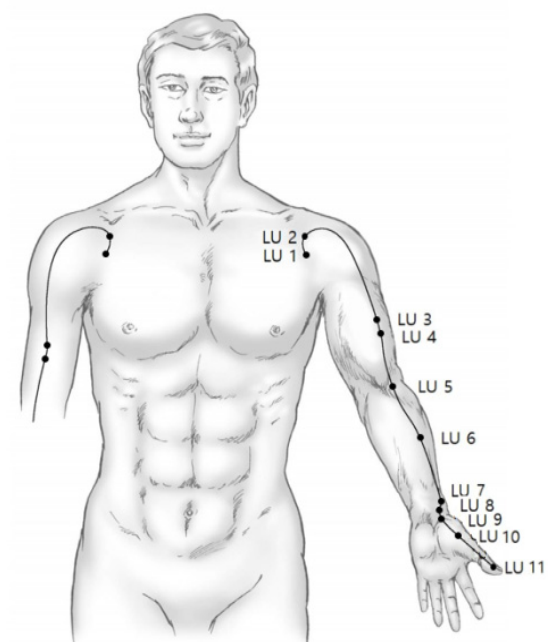

Fig. 8. Pathways and acupoints for the lung meridian of handTaiyin. point, Yuji (LU10) and Shaoshang (LU11). The frequency spectrum at LU1, as shown in Fig. 9(a), shows a narrow bandwidth for each of the frequencies of $1-5,10,30,40$, and $65 \mathrm{MHz}$. This spectrum tendency is similar to all of the other acupoints. Although the intensities of the measured powers, which is the magnitude of the frequency spectrum, are slightly different, the spectrums are basically the same in that the frequency components are the same. It is clear that this similarity of form is significant when we compare these results to those seen in Fig. 4, which shows that the frequency spectrum of the 3 Yin meridians on the hand had quite different basic characteristics due to their locations on different meridians despite their physical closeness. Therefore, these frequency spectrums confirm that measurements taken on eleven acupoints of the same meridian will display similar characteristics. In this way we can only conclude that these points of the lung meridian of hand-Taiyin lie

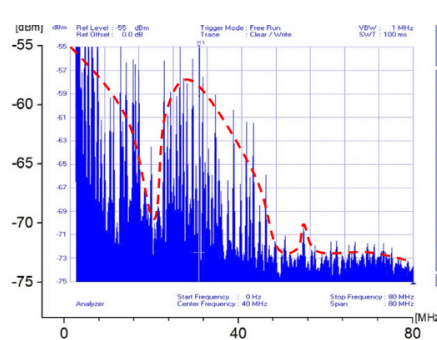

(a)

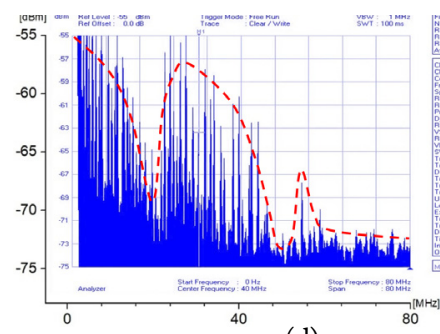

(d)

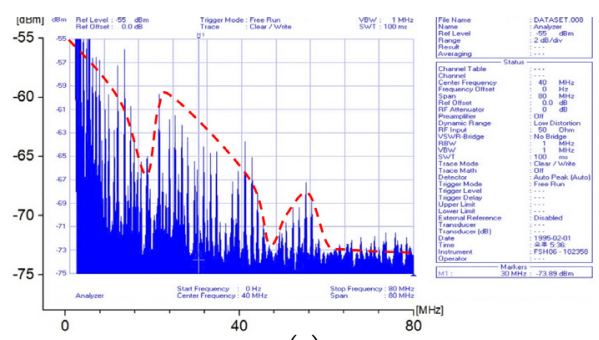

(g)

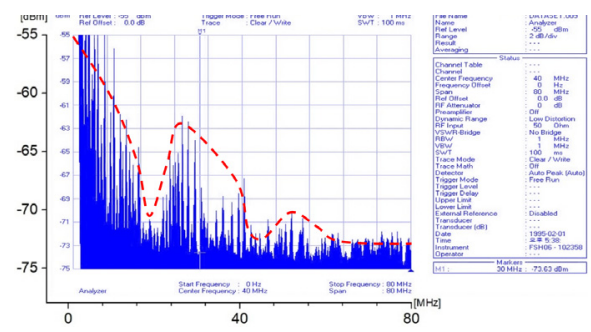

(j)

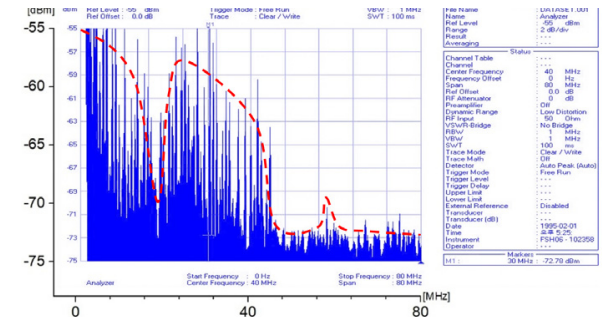

(b)

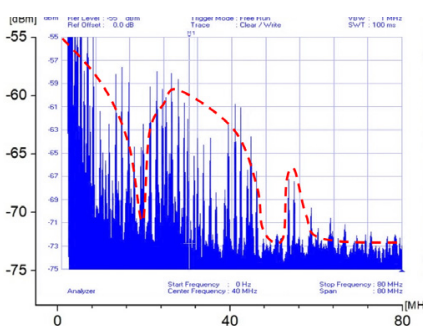

(e)

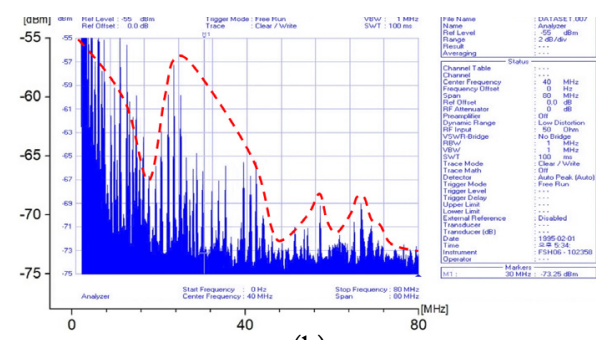

(h)

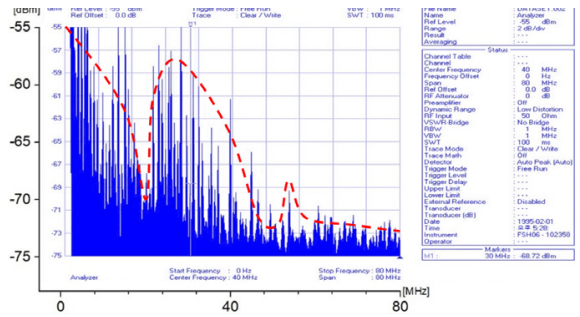

(c)

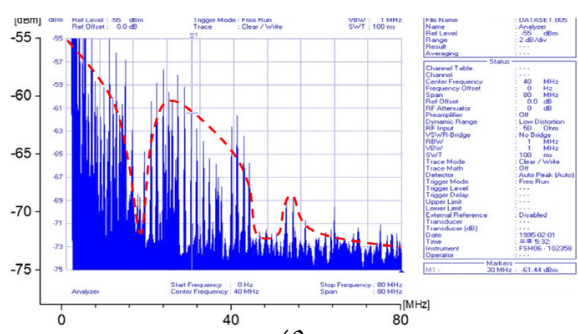

(f)

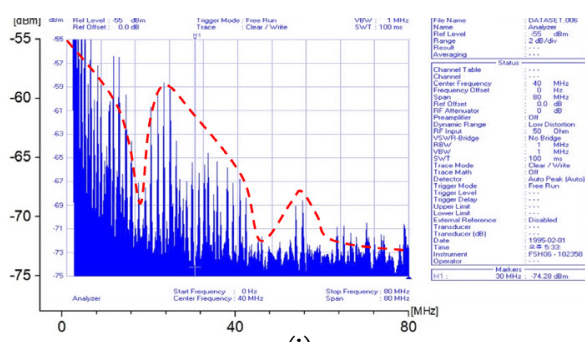

(i)

Fig. 9. Frequency spectrum at acupoints of (a) LU1, (b) LU2, (c) LU3, (d) LU5, (e) LU6, (f) LU7, (g) LU8, (h) LU9, (i) LU10, and (j) LU11 on the lung meridian of hand-Taiyin. 
on a single transmission line of HF electricity that travels throughout the body. The spectrums of LU1 and LU2, which are close to the lungs, have high intensity, which reduces as you move farther away from the lungs. This phenomenon is the same as that seen in transmission line theory of electrical engineering; the signal waveform becomes weaker as it goes farther from the source. This indicates that the $Q i$ energy originates in the lungs and radiates out through the lung meridian.

In this study, a total of 361 acupoints on 14 meridians were measured. The flow through the Yin-Yang meridian in the upper and lower half of the body and also the conception vessel theory was investigated. The upper half Yin-Yang meridians investigated were the lung meridian of hand-Taiyin, shown in Fig. 8, and the large intestine meridian of hand-Yangming, shown in Fig. 10. The liver meridian of foot-Jueyin and the stomach meridian of foot-Yangming for the lower half of the body were also measured.

Fig. 10 shows the acupoints and pathways of the large intestine meridian of hand-Yangming. This meridian has a total of 20 acupoints from Shangyang (LI1), located 0.1 chon ( 1 chon $=$ the length of one horizontal row of finger, about $1.4-1.8 \mathrm{~cm}$ ) from the nail of the forefinger, to Yingxiang (LI20), located on the flank outside the nose.

The measured frequency spectrum for 11 acupoints are showing in Fig. 11: Shangyang (LI1), Sanjian (LI3), He Gu (LI4), Yangxi (LI5), Wenliu (LI7), Shousanli (LI10), Quchi (LI11), Binao (LI14), Jianyu (LI15), Futu (LI18), and Yingxian (LI20).

In Fig. 11, the frequency spectrum intensity of LI1, the point farthest from the large intestine, is smaller than that of LI14 or LI15, which are located nearby. Since the intensity of the spectrum closer to the large intestine becomes greater, the HF electricity at these points is strong. This tells us that $Q i$ comes from the large intestine, which is the corresponding organ to the measured meridian. This gives an especially excellent explanat-

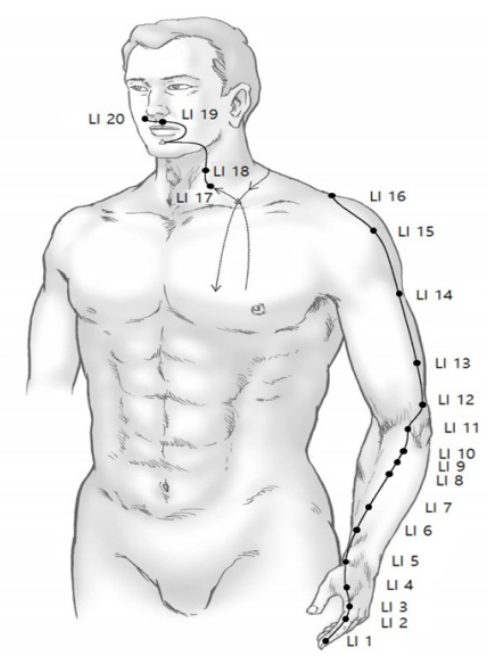

Fig. 10. Pathways and acupoints for the large intestine meridian of hand-Yangming. ion of why the frequency spectrum of LI20, beside the nose, is stronger than at LI1 on the finger. All acupoints on the large intestine meridian of hand-Yangming have frequency components at 1-5, 10, 25, 30, 40, 55, and $65 \mathrm{MHz}$.

Fig. 12 shows the pathways of the liver meridian of footJueyin from LR1 to LR14, and the acupoints on that pathway.

The liver meridian of foot-Jueyin in Fig. 12 depicts a flowing pathway from Dadun (LR1) inside the big toe to Qimen (LR14), the 14th acupoint of a meridian that flows through the foot and leg. The measured frequency spectrum at 9 acupoints of this meridian are shown in Fig. 13. The frequency spectrums are significantly different from the lung meridian of handTaiyin and the large intestine meridian of hand-Yangming, as seen in Figs. 9 and 11, respectively. The frequency spectrums in Fig. 13 show that these acupoints have frequency components at frequencies of $1-5,15,25,30,40,60$, and $70 \mathrm{MHz}$. Although the intensities of the frequency spectrum in Fig. 13(a)-(1) are not similar, the frequency components in the spectrum are the same. Therefore, we can conclude that the HF electricity comes from a single source, in this case the liver, and that the meridian operates as a transmission line that preserves the characteristics of the frequency components. Accordingly, we see a reduction in intensity as we move away from the signal source, just as scientific transmission theory predicts.

Fig. 14 shows the acupoints and pathway of the stomach intestine meridian of foot-Yangming. This meridian has a total of 45 acupoints starting from Chengqi (ST1), located below the eyes, to Lidui (ST45), located at the end of the 2nd toe.

The $Q i$ that flows through the stomach meridian of footYangming originates from the large intestine meridian of handYangming and flows into the spleen meridian of foot-Taiyin. Acupoints on this meridian are used for various treatments of digestive problems, eye disease, problems of the facial nervous system and joint problems. Fig. 15 shows the frequency spectrum at 12 acupoints on this meridian.

In the frequency spectrums of Fig. 15, we can see the intensities of the spectrum change according to the distance from the organ, in this case the stomach, but, unlike the other meridian tested, they are weaker near the stomach and stronger far from the stomach. In further detail, the spectrum intensities for ST4 and ST7, located on the face and shown in Fig. 15(b) and (d), and for ST34 and ST36, located on the leg and shown in Fig. 15(i) and (j), are larger than those at ST21, ST24, and ST25, which are shown in Fig. 15(e), (f), and (g), respectively. This result could be anticipated given the fact that the response to the stomach is greater in the acupoints of the face and feet, which are farther from the stomach, as shown in literature [38] subject as Donguibogam External Appearance. Food stays in the stomach for up to 2-3 hours (if the stomach is empty initially) and then proceeds down to other digestion organs. Therefore, 


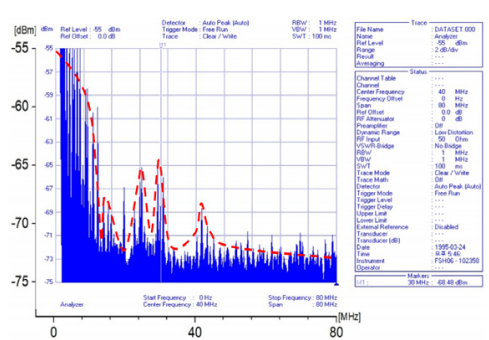

(a)

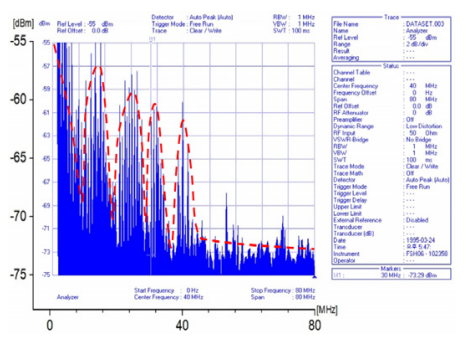

(d)

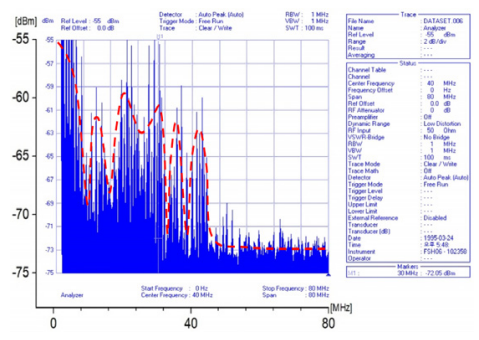

(g)

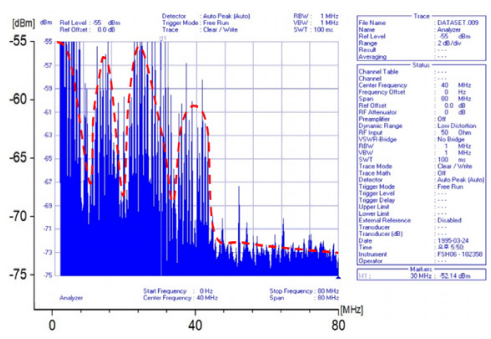

(j)

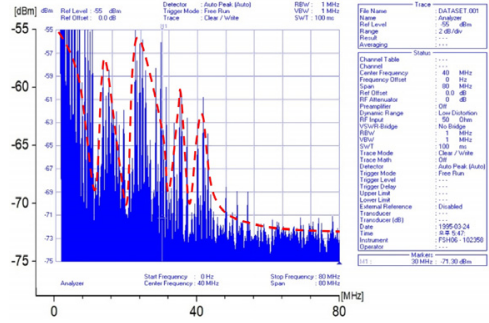

(b)

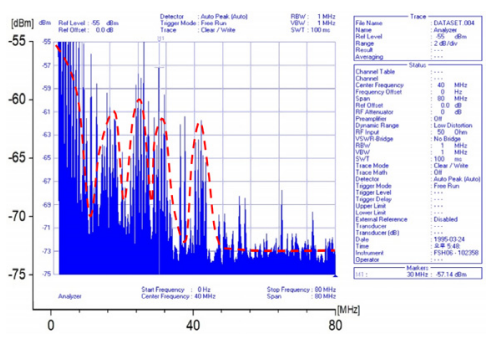

(e)

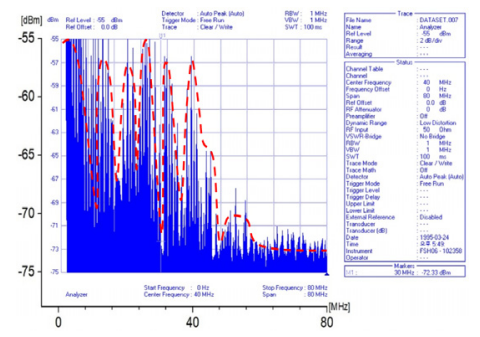

(h)

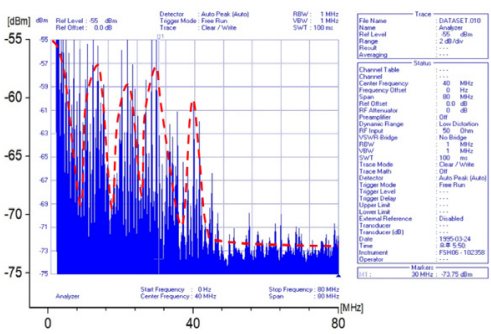

$(\mathrm{k})$

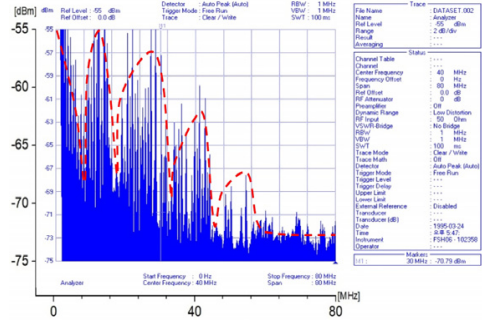

(c)

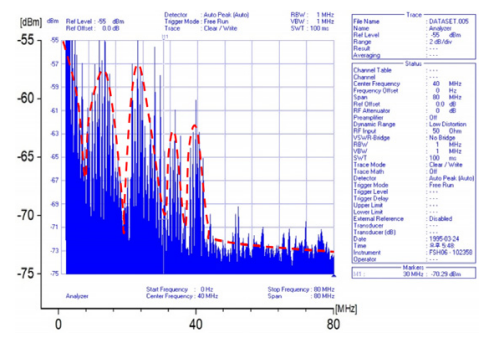

(f)

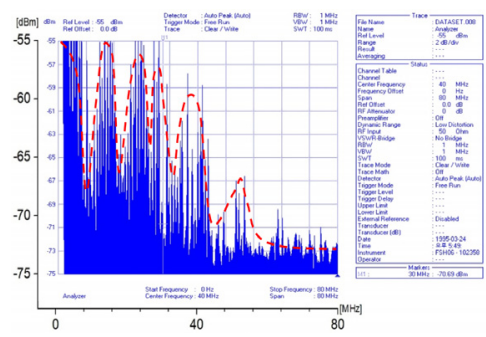

(i)

Fig. 11. Frequency spectrum of acupoints at (a) LI1, (b) LI3, (c) LI4, (d) LI5, (e) LI7, (f) LI10, (g) LI11, (h) LI14, (i) LI15, (j) LI18, and (k) LI20 on the large intestine meridian of hand-Yangming.

we might expect the $Q i$ reaction to appear far away from the stomach. This may be related to lesions in the stomach that relate to abnormalities in the face or limbs. This should be studied in further detail in the future. However, all spectrums look to have the same frequency components: in the $1-30 \mathrm{MHz}$ band, at $40 \mathrm{MHz}$, at $45 \mathrm{MHz}$ and at $65 \mathrm{MHz}$. They have different characteristics than the spectrums of the other meridian, and it is significant that the frequency spectrum of Zusanli (ST36), shown in Fig. 15(j), the most important acupoint in A\&M medicine, has strong and broad frequency band characteristics. Study of the therapeutic effects and how they are related to the frequency spectrums seen at certain acupoints of the various meridians needs to continue in the future.

Finally, the conception vessel that belongs to the eight extraordinary meridian and that controls all Yin meridian is investigated. Due to this, the acupoints of the conception vessel are widely applied in the treatment of diseases along with the acupoints of the 3 Yin meridians of the hand and foot. Fig. 16 shows the pathway and the acupoints of the conception vessel.

The conception vessel has a total of 24 acupoints from Huiyin (CV1) near the anus through the center for the body to Chengjiang (CV24) between the lower lip and jaw. The measured frequency spectrums at 6 acupoints are shown in Fig. 17.

Fig. 17(a)-(f) shows the measured frequency spectrums at 


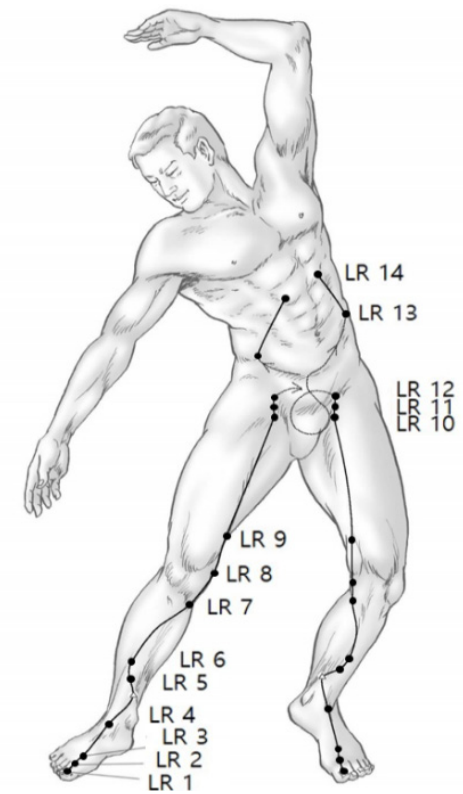

Fig. 12. Pathways and acupoints for the live meridian of footJueyin.

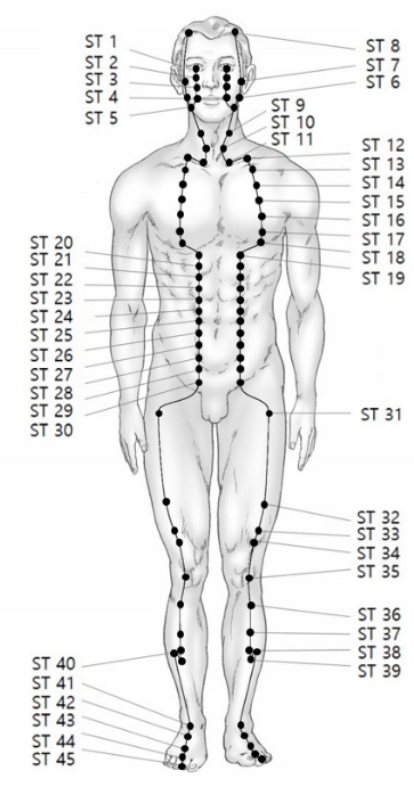

Fig. 14. Pathways and acupoints for the stomach meridian of footYangming.

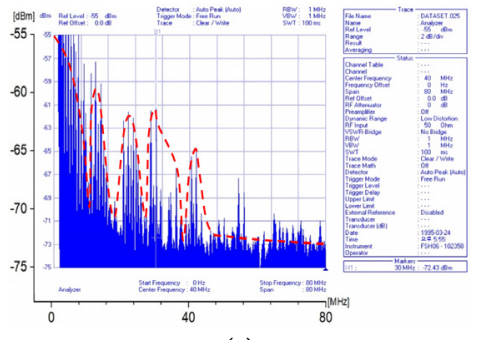

(a)

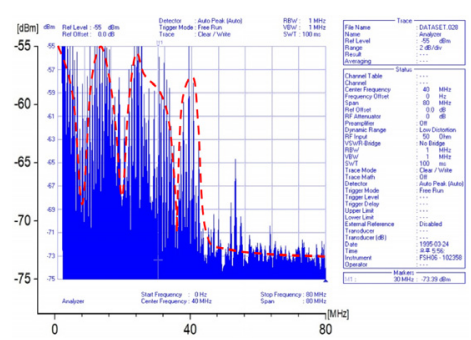

(d)

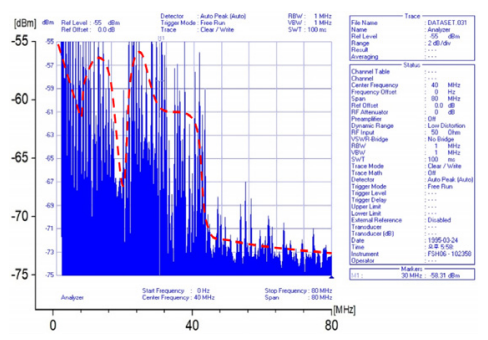

(g)

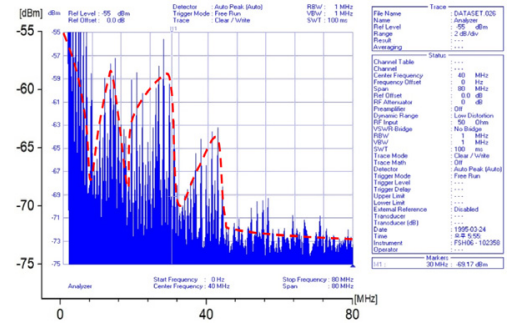

(b)

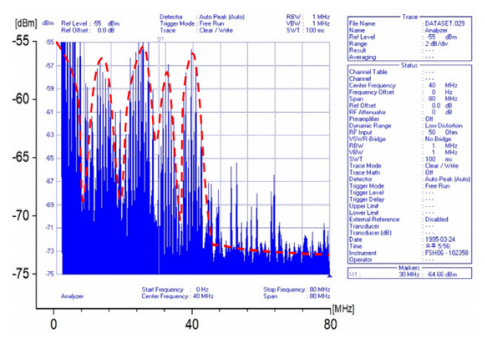

(e)

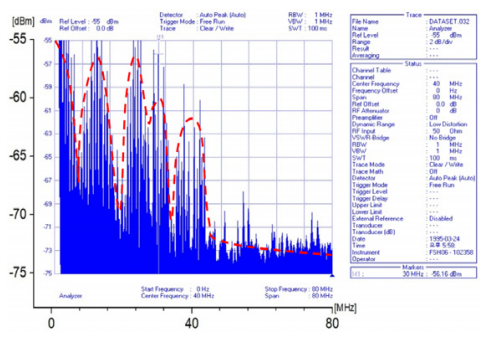

(h)

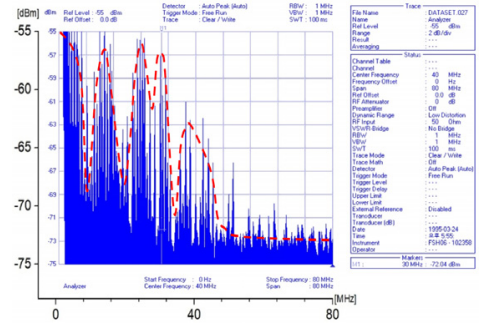

(c)

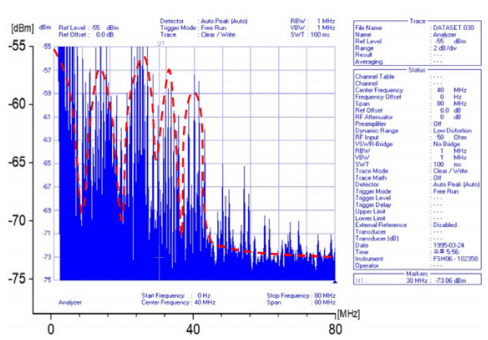

(f)

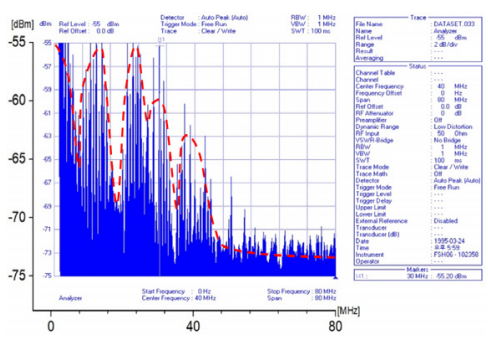

(i)

Fig. 13. Frequency spectrum of acupoints at (a) LR1, (b) LR2, (c) LR3, (d) LR5, (e) LR6, (f) LR8, (g) LR10, (h) LR13, and (i) LR14 on the liver meridian of foot-Jueyin.

Zhongji (CV3), Guanyuan (CV4), Shimen (CV5), Zhongwan (CV12), Juque (CV14), and Shanzhong (CV17) on the concep- tion vessel, repectively. The frequency spectrums are tightly configured so as to be narrow rather than wide. Although the 


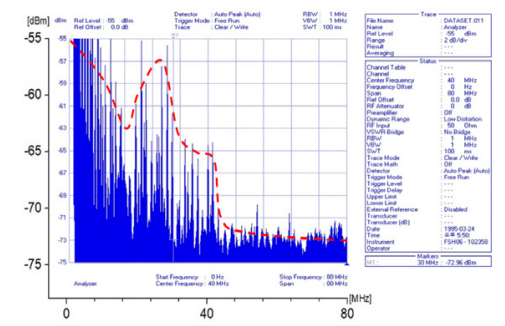

(a)

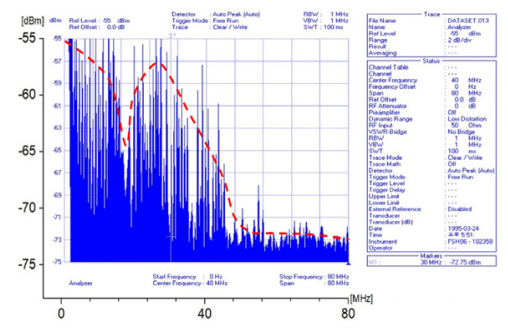

(d)

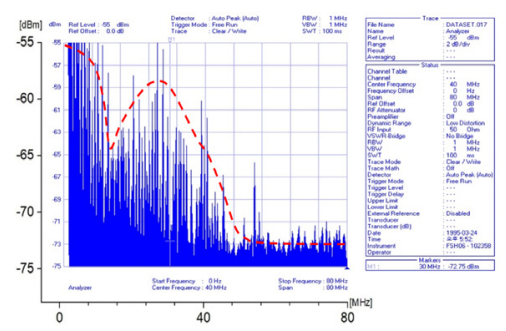

(g)

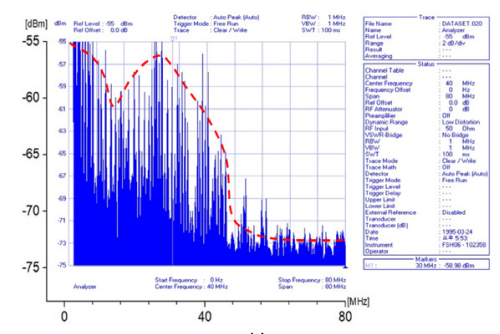

$(\mathrm{j})$

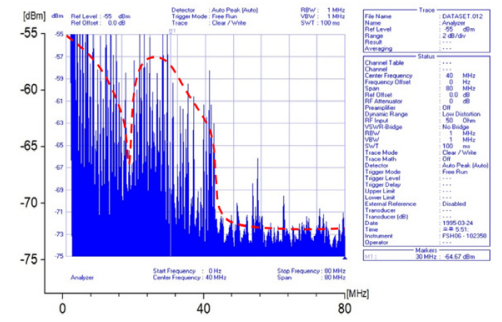

(b)

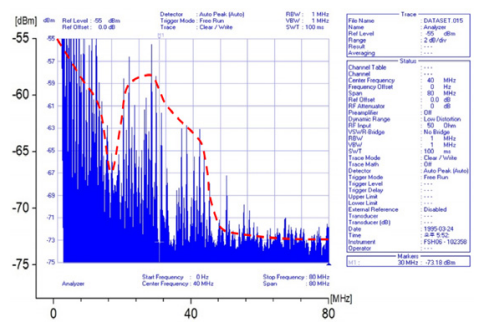

(e)

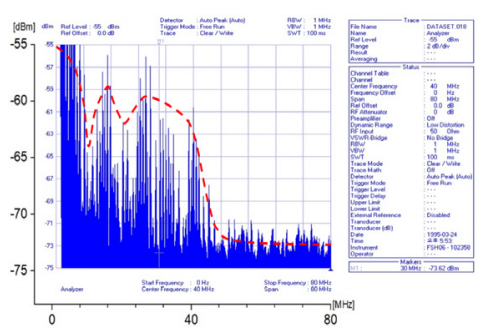

(h)

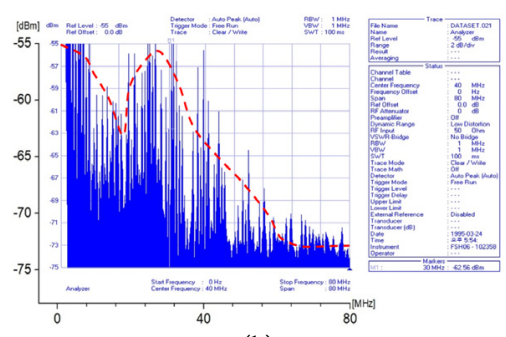

(k)

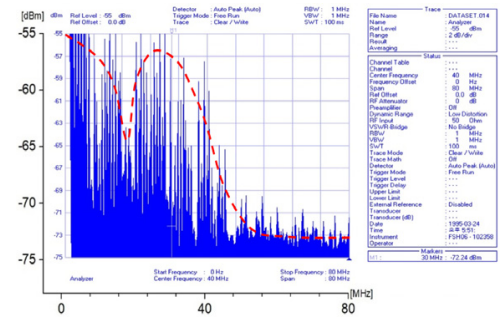

(c)

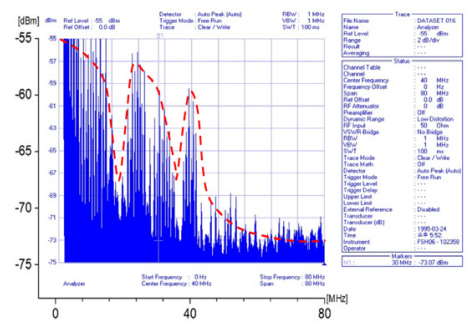

(f)

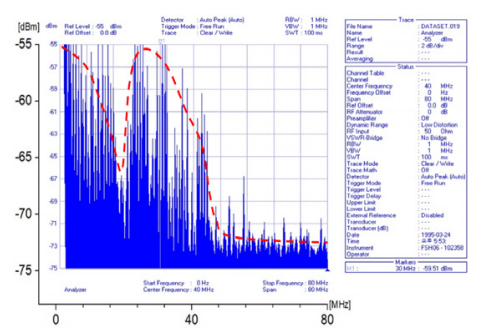

(i)

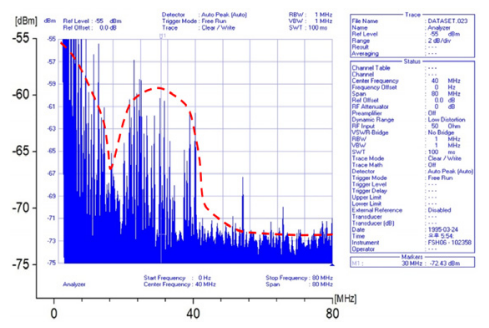

(1)

Fig. 15. Frequency spectrum of acupoints at (a) ST2, (b) ST4, (c) ST6, (d) ST7, (e) ST21, (f) ST24, (g) ST25, (h) ST28, (i) ST34, (j) ST36, (k) ST40 and (1) ST44 on the stomach meridian of foot-Yangming.

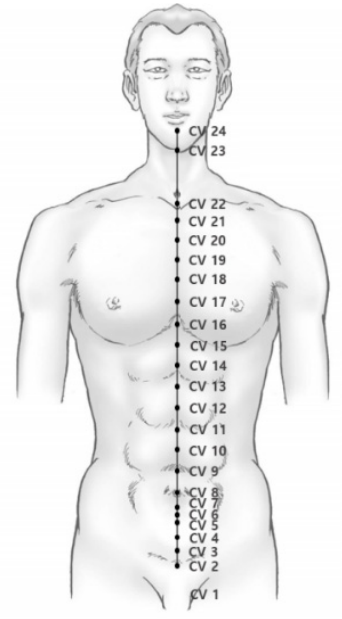

Fig. 16. Pathway and acupoints for the conception vessel. intensities of the spectrum are not large, they all have the frequency components found at the 3 Yin meridians of the hand and foot. This explains how the conception vessel has the ability to control the entire Yin meridian. If we examine the frequency spectrum in Fig. 17, it is absolutely clear that the acupoints on the conception vessel have frequency components that are all the same. In addition, the intensities are almost constant, which is explained neatly by the fact there is no corresponding organ of the conception vessel.

\section{CONCLUSION}

In this study, we revealed, by measurement of frequency spectrums, the existence of HF electricity flowing through the very 


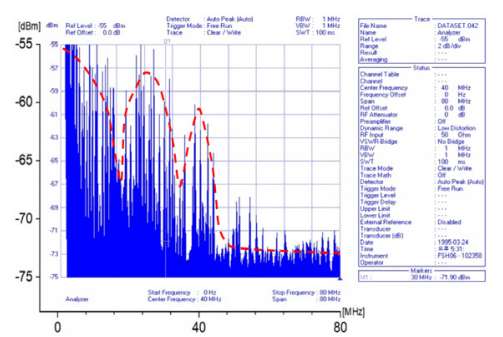

(a)

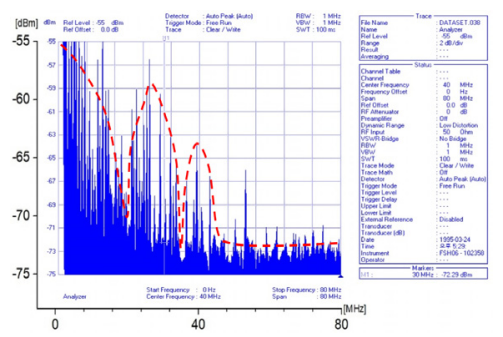

(d)

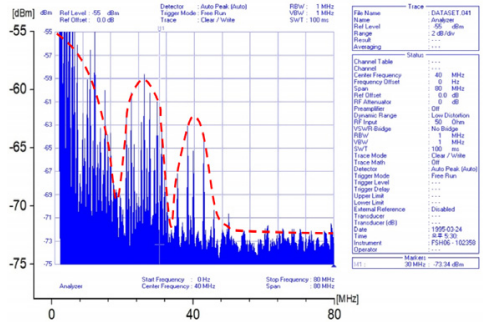

(b)

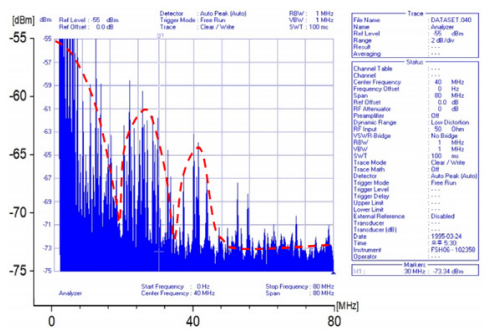

(e)

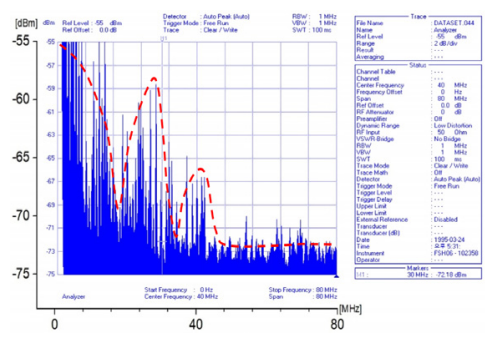

(c)

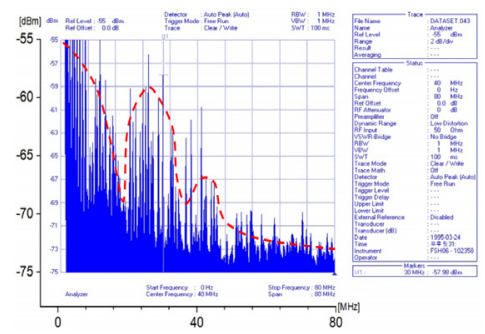

(f)

Fig. 17. Frequency spectrum of acupoints at (a) CV3, (b) CV4, (c) CV5, (d) CV12, (e) CV14, and (f) CV17 on the conception vessel.

particular acupoints that are widely used in acupuncture and moxibustion. Analysis of the measured frequency spectrums leads naturally to the truth that there must be some kind of transmission line located exactly along the lines of the meridian known to TCM. The acupoints used for acupuncture treatment and therapy were found to contain various frequencies between $1 \mathrm{MHz}$ and $80 \mathrm{MHz}$ with intensities of -70 to $-60 \mathrm{dBm}\left(10^{-10}\right.$ to $10^{-9} \mathrm{~W}$ ). Different spectrums measured at each primary acupoint of the 3 Yin meridians of the hand, 3 Yang meridians of the hand, 3 Yin meridians of the foot and 3 Yang meridians of the foot tell us that these 12 meridians have their own HF electricity flow. The same frequency spectrum characteristics found at the acupoints along the meridian show that the same HF current flow was being measured at various points along the meridian. From this we must conclude that the meridian operates just like transmission lines in electrical engineering. Thus, the meridian and acupoints used in oriental medicine coming from ancient times were verified through our investigation of spectrum measurements. The results of this study show that the changes in the $\mathrm{HF}$ spectrum at these acupoints as the result of a related disease is to be expected, and these kind of measurements will become a cornerstone in the process of clarifying the mechanism of A\&M treatment. In addition, finding the optimal combination of acupoints in the treatment of various pathologies has exciting potential as a future research topic.

This work was supported by the Soonchunhyang University Research Fund. The authors would like to express their sincere thanks to Director SangJun Lee of HCT Co. Ltd., Korea, who kindly supported the measurement site and Prof. Hyun Yoo of the Korea Institute of Traditional Acupuncture and Moxibustion for useful suggestions on oriental medicine theory.

\section{REFERENCES}

[1] N. Kim, Meridian and Acupuncture, 2nd ed. Seoul, Korea: Institute of Authentic Acupuncture and Moxibustion, 2015.

[2] J.J. Tsuei, "The science of acupuncture-theory and practice," IEEE Engineering in Medicine and Biology Magazine, vol. 15, no. 3, pp. 52-57, 1996.

[3] N. Kim, Theory and Practice of Moxibustion, 2nd ed. Seoul, Korea: Institute of Authentic Acupuncture and Moxibustion, 2015.

[4] W. A. Lu, J. J. Tsuei, and K. G. Chen, "Preferential direction and symmetry of electric conduction of human meridians: bilaterally symmetrical acupoints provide better conductance for a better "connection," IEEE Engineering in Medicine and Biology Magazine, vol. 18, no. 1, pp. 76-78, 1999.

[5] N. Kim, Pathology and Etiology, 2nd ed. Seoul, Korea: Institute of Authentic Acupuncture and Moxibustion, 2015.

[6] N. Kim, Diagnostics of Acupuncture and Moxibustion, 2nd ed. Seoul, Korea: Institute of Authentic Acupuncture and Moxibustion, 2014.

[7] Z. M. Wang, Y.H. Tan, and M. Y. Su, "Analysis of acupuncture point signal using wavelet transform and higher-order statistical method," in Proceedings of 2009 International Conference on Apperceiving Computing and Intelligence Analysis, Chengdu, China, 2009, pp. 18-21.

[8] K. G. Chen, "Biological implication of electrical properties of acupuncture meridians," in Proceedings of the 20th Annual International Conference of the IEEE Engineering in Medicine and Biology Society, Hong Kong, China, 1998, pp. 10861087.

[9] K. G. Chen, "Electrical properties of meridians," IEEE En- 
gineering in Medicine and Biology Magazine, vol. 15, no. 3, pp. 58-63, 1996.

[10] S. Y. Chun, U. H. Ji, and H. T. Nagle, "Observations of the current change on acupuncture spots at the meal time," in Proceedings of the 17th IEEE Symposium on ComputerBased Medical Systems, Bethesda, MD, 2004, pp. 490-495.

[11] M. H. Yeh, H. L. Luo, N. W. Lin, Z. Y. Chen, and C. C. Yeh, "A study on electrical properties of acupuncture points in allergic rhinitis," in Proceedings of 2012 IEEE 14th International Conference on e-Health Networking, Applications and Services (Healthcom), Beijing, China, 2012, pp. 82-87.

[12] O. G. Martinsen, S. Grimnes, L. Morkrid, and M. Hareide, "Line patterns in the mosaic electrical properties of human skin: a cross-correlation study," IEEE Transactions on Biomedical Engineering, vol. 48, no. 6, pp. 731-734, 2001.

[13] K. Saputra, "Biophysics, biomolecular and radionuclide tracing technique to explain signal transduction profile of acupuncture points," in Proceedings of the 2nd International Conference on Bioelectromagnetism (Cat. No. 98TH8269), Melbourne, Australia, 1998, pp. 95-96.

[14] J. J. Tsuei, F. M. K. Lam, and P. Chou, "Clinical applications of the EDST," IEEE Engineering in Medicine and Biology Magazine, vol. 15, no. 3, pp. 67-75, 1996.

[15] H. S. Myoung, H. S. Choi, K. J. Lee, and Y. H. Lee, "Development of acupoints discrimination system using electric properties of acupoints and non-acupoints," in Proceedings of 2008 30th Annual International Conference of the IEEE Engineering in Medicine and Biology Society, Vancouver, Canada2008, pp. 4924-4927.

[16] M. Cohen, C. Behrenbruch, and I. Cosic, "Is there a link between acupuncture meridians, Earth-ionosphere resonances and cerebral activity?," in Proceedings of the $2 n d I^{n-}$ ternational Conference on Bioelectromagnetism (Cat. No. 98TH8269), Melbourne, Australia, 1998, pp. 173-174.

[17] Q. Yang, Z. Gu, Z. Xu, and Q. Chen, "Clinical research on rule of acupoint heat-sensitization within patients suffer Bell's palsy," in Proceedings of 2012 IEEE International Conference on Bioinformatics and Biomedicine Workshops, Philadelphia, PA, 2012, pp. 413-417.

[18] Q. Fang, R. Bedi, B. Ahmed, and I. Cosic, "Comparison of skin resistance between biological active points of left and right hands with various contact pressures," in Proceedings of the 26th Annual International Conference of the IEEE Engineering in Medicine and Biology Society, San Francisco, CA, 2004, pp. 2995-2998.

[19] M. Reichmanis, A. A. Marino, and P. O. Becker, "Electrical correlates of acupuncture points," IEEE Transactions on Biomedical Engineering, vol. 22, no. 6, pp. 533-535, 1975.

[20] Suhariningsih and H. Kanai, "Observation of human impedance at $50 \mathrm{kHz}$ for analysis of acupuncture points," in Proceedings of the 2nd International Conference on Bioelectromagnetism (Cat. No. 98TH8269), Melbourne, Australia, 1998.

[22] Y. H. Ryu, S. J. Cho, S. H. Lee, S. B. Lee, K. H. Choi, and S. M. Choi, "The biopotential of acupuncture points and its standard error," in Proceedings of 2011 IEEE MTT-S International Microwave Workshop Series on Intelligent Radio for Future Personal Terminals, Daejeon, Korea, 2011, pp. 1-2.

[22] M. Kukucka, Z. Krajcuskova, V. Stopjakova, and D. Durackova, "The influence of the amplitude of driving signal in measurement of the active points on human skin," in Proceedings of 2013 23rd International Conference Radioelektronika, Pardubice, Czech Republic, 2013, pp. 166-171.

[23] M. Kukucka and Z. Krajcuskova, "Various effects in voltage chart measurement of active points on skin," in Proceedings of the 21st International Conference Radioelektronika, Brno, Czech Republic, 2011, pp. 1-4.

[24] J. Yuan, J. Chen, L. Huang, F. Xu, M. Q. Yang, S. Yan, G. Li, and Z. Yang, "Visualized analysis of incomplete TCM meridian conductance data," in Proceedings of 2016 IEEE International Conference on Bioinformatics and Biomedicine (BIBM), Shenzhen, China, 2016, pp. 1406-1408.

[25] O. G. Martinsen, S. Grimnes, L. Morkrid, and M. Hareide, "Line patterns in the mosaic electrical properties of human skin-a cross-correlation study," IEEE Transactions on Biomedical Engineering, vol. 48, no. 6, pp. 731-734, 2001.

[26] C. Huang, Y. Wang, X. Lu, and Z. Wang, "Study on DC resistance and AC steady-state characteristics of the Pericardium Meridian on skin surface," in Proceedings of International Conference on Information Science and Technology, Nanjing, China, 2011, pp. 1335-1338.

[27] K. S. Lee and Y. S. Kim, "Study of the thermal differences on the meridian points used to the cold hypersensitivity," in Proceedings of the 19th Annual International Conference of the IEEE Engineering in Medicine and Biology Society, Chicago, IL, 1997, pp. 634-636.

[28] J. B. Kim, S. M. Lee, C. Choi, Y. B. Kim, and G. Yoon, "Propagated photon detection at PC6 irradiating along the pericardium meridian," in Proceedings of the 4th Pacific Rim Conference on Lasers and Electro-Optics (Cat. No. 01TH8557) Technical Digest, Chiba, Japan, 2001.

[29] H. Q. Yang, S. S. Xie, X. L. Hu, L. Chen, and Z. K. Lu, "Infrared spectrum visualizing human acupoints and meridian-like structure," in Proceedings of 2006 International Symposium on Biophotonics, Nanophotonics and Metamaterials, Hangzhou, China, 2006, pp. 54-56.

[30] J. Ying, X. Shen, H. Li, G. Ding, H. Chen, C. Sun, and S. $\mathrm{Hu}$, "Study on body surface infrared radiation spectrum of points of patients with hyperplasia of mammary gland," in Proceedings of 2008 IEEE International Symposium on IT in 
Medicine and Education, Xiamen, China, 2008, pp. 587-591.

[31] L. I. Lisitsyna, L. G. Navrotsky, T. M. Starovoytova, A. A. Kamardin, S. V. Belavskaya, and A. A. Blokhin, "Effect of transfer of light energy on acupuncture meridians," in Proceedings of 2017 18th International Conference of Young Specialists on Micro/Nanotechnologies and Electron Devices (EDM), Erlagol, Russia, 2017, pp. 616-618.

[32] H. M. Sathyendra, J. E. Chan, K. U. Sivaprasad, and J. R. LaCourse, "Transmission line modeling for acupuncture nodal therapy," in Proceedings of 2003 IEEE 29th Annual Proceedings of Bioengineering Conference, Newark, NJ, 2003, pp. 126-127.

[33] S. Chang, "The rationale behind a reticular meridain model for Chinese acupuncture," in Proceedings of 2010 4th International Conference on Bioinformatics and Biomedical Engineering, Chengdu, China, 2010, pp.1-4.

[34] T. Son and Y. Yoo, "Spectrum measurement of the electric

Taeho Son

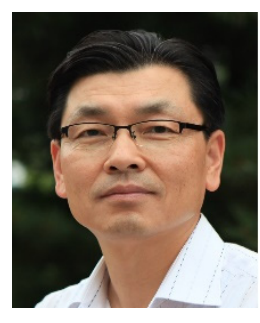

received a B.S. degree, a M.S. degree and a Ph.D. degree from Hanyang University, Korea, in 1979, 1986, and 1990, respectively. He graduated from the Academy of Korean Authentic Acupuncture and Moxibustion (AKAAM), Korea, in 2018 and became a registered acupuncture and moxibustion therapist. From 1981 to 1982, he was a researcher with the Radar Center at Ferranti Company, Edinburgh, Scotland. He worked for Gold Star Precision Company as a team leader of the Radar and RF System Division from 1978 to 1987. Since 1990, he has been a professor in the Department of IT Engineering at Soonchunhyang University, Korea. He was a chairperson at the Korea Institute of Intelligent Transportation System (KITS) in 2011. He was a technical advisor at several companies concerned with antennas for RF systems and mobile phones and is working on advanced antenna engineering at Skycross Korea Company, Korea. Currently, his research is mainly focused on the design of various antennas for mobile communication with the $5 \mathrm{G}$ network and electronic systems for vehicles, and he is also interested in the area of electrical engineering principles, especially regarding the effects of acupuncture and moxibustion. He has authored or co-authored over 310 papers in journals and conference proceedings. Dr. Son is a senior member of IEEE and a member of KIEES, IEIE, KICS and KITS. signal at the human acupoints for the acupuncture and moxibustion," in Proceedings of 2017 Microwave and Antenna Propagation Conference Digest, 2017.

[35] T. Son, Y. Yoo, and M. Lee, "Proof of the oriental medicine meridian flowing by high frequency spectrum," in Proceedings of 2017 Fall Conference Digest of Korea Institute Electromagnetic Engineering and Science, 2017.

[36] S. J. Lee, W. S. Yoon, and S. M. Han, "Planar directional beam antenna design for beam switching system applications," Journal of Electromagnetic Engineering and Science, vol. 17, no. 1, pp. 14-19, 2017.

[37] World Health Organization, WHO Standard Acupuncture Point Locations in the Western Pacific Region. Manila, Philippine: World Health Organization; 2008.

[38] H. Jun, Donguibogam External Appearance. Seoul, Korea: Kngl Press Co., 2014.

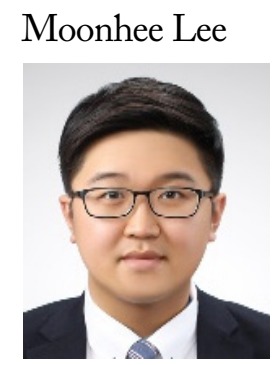

received his B.S. and M.S. in IT engineering from Soonchunhayng University, Korea, in 2015 and 2017, respectively. He is currently pursuing his Ph.D. in IT engineering at Soonchunhayng University, Korea. His research interests include antennas for the RF system and the vehicle, antennas for base stations and mobile phones, and needle antennas for MW ablation. 


\section{Choolhyeong Lee}

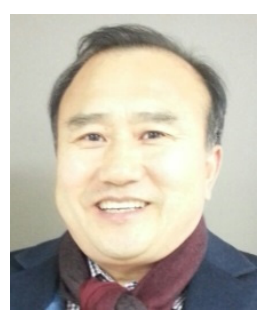

was born in Gangwon Province, Korea, in November 1959. He obtained a B.S. degree from Gangneung-Wonju National University, Korea, in 1985, and a M.S. degree of Integrated Medicine from Cha University, Korea, in 2018. He graduated from the Academy of Korean Authentic Acupuncture and Moxibustion (AKAAM), and became a registered acupuncture and moxibustion therapist in 2003. He took the International Acupuncture Doctor qualification by the World Federation of Chinese Medicine Societies (WFCMS), China, in 2011. Since 2007, he has been a professor at AKAAM.

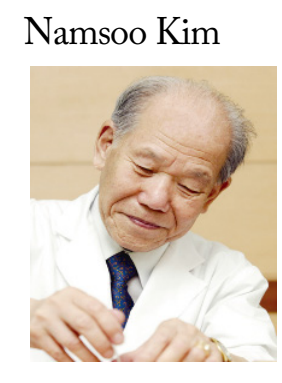

was born in Gwangsan-gun, Jeolla Province, Korea, in 1915. He obtained Honorary Doctorates of Oriental Medicine and Natural Healing from South Baylor University and Rodland University, USA in 2009 and 2012, respectively. Since he opened his acupuncture and moxibustion clinic in 1943, he has been treating and practicing for 77 years. He was a professor of the Beijing University of Chinese Medicine, China and a professor of Natural Medicine at the Green University, Korea. Now he is a member of the Korea Acupuncture Association, a $\mathrm{CEO}$ and a professor at the Academy of Korean Authentic Acupuncture and Moxibustion (AKAAM), Korea. Also, he is an executive committee member, an education committee member, and an acupuncture and a moxibustion examination committee member of the World Federation of Acupuncture-Moxibustion Societies (WFAS), a member and an executive committee member of the World Association of Chinese Medicines, a Korean representative in the event of an international bedding accident, a chairman of the legislative promotion committee, and a volunteer director of the Korean Acupuncture Association. He has written many curriculum books for the Seoul National School for the Blind and the AKAAM. 